\title{
Conduction Velocity in Various Regions of the Ureter
}

\author{
By \\ Makoto Kobayashi \\ From the Department of Physiology, School \\ of Medicine, Hiroshima University, Hiroshima; \\ Director: Prof. H. I ris a wa \\ (Received for publication, April 7, 1964)
}

\begin{abstract}
Conduction velocity of excitation in various regions of the cat ureter was studied through the simultaneous recordings of five action potentials of the ureter by using of the multiple electrodes. The conduction velocity was very slow in the renal pelvis where the pacemaker was located, i.e., about $2 \mathrm{~mm} / \mathrm{sec}$ on an average. In the proximal region of the ureter, the velocity gradually increased with distance from the pelvis, while the velocity became constant (about $200 \mathrm{~mm} / \mathrm{sec}$ ) in the regions more than about $15 \mathrm{~mm}$ away from the renal end of the ureter. It was concluded that the conduction velocity of excitation was significantly different between the pacemaker region and the follower tissue of the ureter.
\end{abstract}

For the computation of the conduction velocity of excitation, it is generally assumed that the excitation travels with a constant velocity throughout the tissue concerned. In the homogeneous preparation such as myelinated nerve fiber, this assumption may be applicable with less errors, but in smooth muscles the situation appears to be more complex. In the ureter smooth muscle, for instance, an excitation spreads three dimensionally through the network of the circular and the longitudinal muscle fibers, therefore the whole ureter does not appear to be a uniform tissue in structure for conduction of excitation. Previous studies on the conduction velocity in ureter muscle ${ }^{1-4)}$ seem to have neglected the differences of velocity among various regions of the ureter tissue, and have assumed that the excitation travels with a constant velocity throughout the tissue. It seems desirable to re-examine this fundamental assumption by using ureter muscle.

The procedure for preparing the cat ureter was almost the same as previously described ${ }^{5}$, however in the present experiment special attention was paid to preserve the renal pelvis and the calyces as much as possible. To obtain such a preparation the ureter was isolated together with the kidney, and the renal 
parenchyma was carefully removed under the dissection microscope to isolate the renal pelvis and the calyces. The preparation was then placed in a bath controlled at $37^{\circ} \mathrm{C}$, and oxygenated Ringer-Krebs solution was perfused continuously. Since the handling of the preparation may cause a change in the muscle dimension which in turn may produce an experimental error on the measurement of conduction velocity, the ureter was kept at its original length by placing marks with Indian ink at $1 \mathrm{~cm}$ intervals in situ, and by keeping the marked intervals at their original length while the ureter is in the bathing solution. The extracellularly recorded action potential of ureter was used as an indicator of the arrival of an excitation. To obtain the potentials, four external electrodes were employed as reference electrodes of the unipolar lead. They were made contact with the muscle by thin cottonwicks of about $500 \mu$ in diameter at the muscle. The indifferent electrodes were made up with larger wicks and placed in remote places away from the preparation in the bath. The potentials were amplified with four channel CR amplifiers of a time constant of $0.3 \mathrm{sec}$. In the renal pelvis the potential was too small to be recorded by these surface electrodes, therefore, the routine glass ultramicroelectrode was employed to obtain the potential from this region, and the potentials were amplified with a high input impedance negative capacity amplifier. The multi-channel inkwriting recorder was used for recording these action potentials.

Bozler ${ }^{6)}$, in his pioneer work of ureter muscle, found the pacemaker of the ureter in its extreme renal end. In the isolated preparation with the intact renal pelvis and the calyces, the spontaneous contractions were found to initiate from the border of the renal pelvis and the calyces, and the frequency was $4-8 / \mathrm{min}$. At the top of Fig. 1, a schematic drawing of the renal pelvis and the ureter is shown where the positions of one microelectrode (A) and four wick electrodes (B-E) are indicated. Tracing $A$ of this figure illustrates the potential led off through a microelectrode at the pacemaker region. Although it is not certain whether this is an intracellularly recorded pacemaker potential or not, the onset of the potential was slightly earlier than that of the mechanical contraction, so it was assumed to coincide with the beginning of excitation at this region. Tracings B-E are the surface action potentials recorded from four different sites shown in the top illustration. It is noteworthy that the amplitude and the rising rate of the action potentials were smaller as the recordings were taken closer to the renal pelvis. Sometimes, oscillatory potentials superimposed on the plateau were observed in the renal end of the ureter.

The conduction velocity in the renal pelvis was obtained by the distance between the two electrodes, $\mathrm{A}$ and $\mathrm{B}$, divided by the time interval between the onset of potential $A$ and the peak of potential $B$, the latter was assumed to show the approximate time when a wave passed directly under the electrode $B$; the velocity was found to be very slow, i.e., about $2 \mathrm{~mm} / \mathrm{sec}$ on an average. The 
velocity in the ureter was measured by the distance between two electrodes divided by the time interval between peaks of the two action potentials, and the average value of each $3 \mathrm{~mm}$ separation was gradually increased from the proximal region of the ureter to the distal region, such as from $6,7.5$, and then $12 \mathrm{~mm} / \mathrm{sec}$.

The distances between the electrodes were varied between 1 and $20 \mathrm{~mm}$, and whenever the position of the electrodes was changed, simultaneous recordings of action potentials were made. Thus, the conduction time of excitation throughout the ureter was measured. The result is illustrated in Fig. 2, and each circle of this figure shows the average value of 8 to 13 measurements. The conduction time in the renal pelvis was about $3 \mathrm{sec}$, and in the figure it is shown by the dotted curve obtained by means of interpolation. In the proximal region of the ureter the slope of the curve gradually decreased with distance from the pelvis, i.e., the conduction velocity gradually increased within the ureter. In the regions more than about $15 \mathrm{~mm}$ away from the renal end of the ureter the line became straight, which indicates the uniformity of the conduction velocity. In the particular case

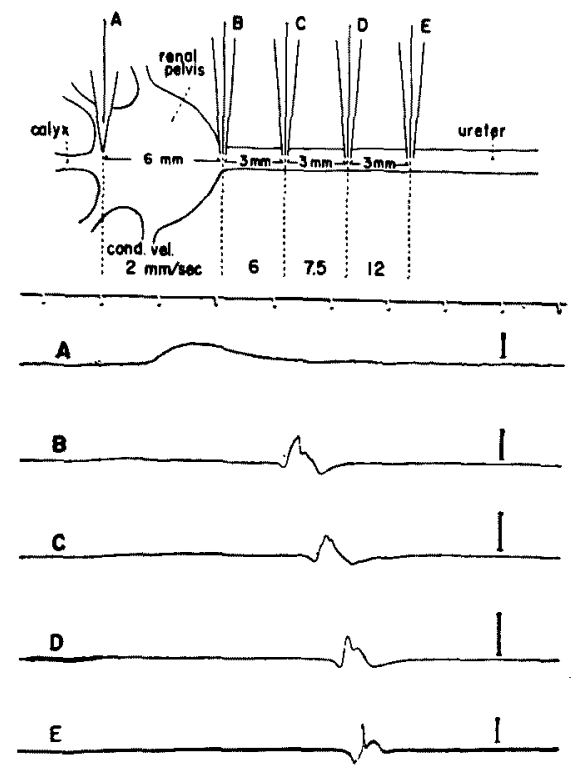

Fig. 1

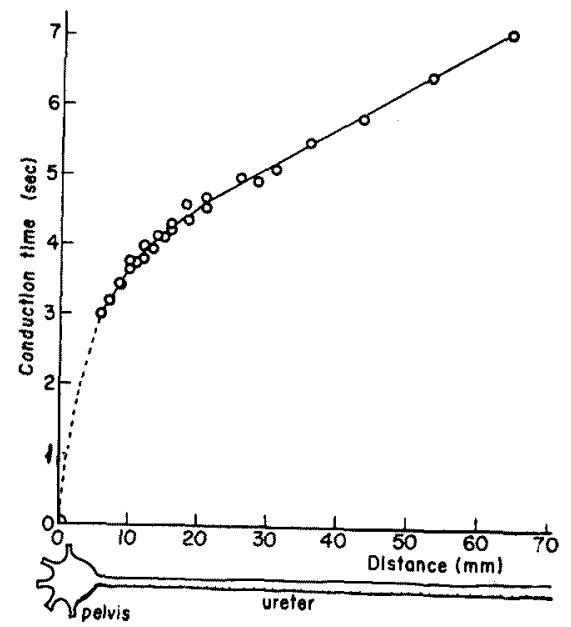

Fig. 2

Fig. 1. Action potentials simultaneously recorded from the renal pelvis and four different sites of the ureter. Time interval 1 sec. Voltage calibration in A represents $20 \mathrm{mV}$, in B $100 \mu \mathrm{V}$, in $\mathrm{C} 400 \mu \mathrm{V}$, and in D and E $500 \mu \mathrm{V}$. See text in detail.

Fig. 2. Conduction time through the renal pelvis and the ureter. Ordinate: time of arrival of excitation to the recording site. Time zero represents the time of the onset of the potential recorded from the pacemaker region, Absicssa: distance between the pacemaker region and the recording site. 
shown in Fig. 2, it was $18.2 \mathrm{~mm} / \mathrm{sec}$. The same experiments were repeated using several ureters and similar results were obtained except for a slight difference in the absolute value of conduction velocity.

From the above results it can be said that the conduction velocity of excitation is significantly different between the renal pelvis and the ureter, and that it is also not uniform in the ureter tissue. Previous studies on the conduction velocity of ureter ${ }^{1-4)}$ appear to have been made by using the ureter tube away from the renal pelvis where a uniform conduction velocity can be safely assumed. But, if the proximal region of the ureter is included in the measurement of the conduction velocity, a uniform conduction cannot be assumed. There may be two possibilities for the causes of the slow conduction velocity in the renal pelvis and the proximal region of the ureter. 1) Since the conduction of excitation in smooth muscle is considered to take place by the electrical transmission from cell to cell7,8), the conduction velocity may depend on the excitability of individual cell membrane and the nature of intercellular barriers. The first possibility can therefore be that these two factors may be different among the various regions of ureter and pelvis. 2) Another possibility that can be considered is the difference of the dimension of the muscle fibers. If the muscle fibers are smaller and shorter in the renal pelvis and in the proximal region of the ureter than the rest of the ureter, there may be more cell junctions across which the excitation travels, and therefore a slower conduction velocity can be expected. The extreme difficulty of inserting the microelectrode into the cell of either the pelvis or the proximal region of the ureter, and the facts that by the large wick electrode the action potential could not be recorded in the pelvis, and that sometimes the oscillatory potentials were obtained in the renal end the ureter, may support this possibility. Furthermore, the arrangement of muscle fibers in the cat ureter may be different in various regions as described in the waterbuffalo ureter ${ }^{9}$. A comparison of the pacemaker of the ureter with that of the vertebrate myocardium, which has a slow conduction velocity ${ }^{10}$, is under progress by recording the electrical activities from four different sites of the renal pelvis.

Acknowledgment is made to Miss S. Hirahara for her assistance in the preparation of the manuscript.

This work was supported by grants from the U.S. Department of Army through its Far East Research Office, from the National Heart Institutes (HE 06968-02), and from the Abbott Laboratories to H. Irisawa, in whose laboratory the work was done.

\section{References}

1) Sleator, W. \& Butcher, H.R., Amer. J. Physiol., 1955, 180, 261.

2) Prosser, C.L., Smith, C.E. \& Melton, C.E., ibid., 1955, 181, 651.

3) Burnstock, G. \& Prosser, C.L., ibid., 1960, 199, 553.

4) Ichikawa, S. \& Ikeda, O., Jap. J. Physiol., 1960, 10, 1. 
5) Kobayashi, M. \& Irisawa, H., Amer. J. Physiol., 1964, 206, 205.

6) Bozler, E., ibid., 1942, 136, 543.

7) Bülbring, E., Burnstock, G. \& Holman, M.E., J. Physiol., 1958, 142, 420.

8) Prosser, C.L., Physiol. Rev., 1962, 42, Suppl. 5, 193.

9) Gould, D.W., Hsieh, A.C.L. \& Tinckler, L.F., J. Physiol., 1955, 129, 425.

10) Paes de Carvalho, A., de Mello, W.C. \& Hoffman, B.F., Amer.J. Physiol., 1959, 196, 483. 